\title{
Heat transfer and modeling hollow cathode material structural state of vacuum plasmatron
}

\author{
Dutova O.S. \\ Dept. of Electrotechnology \\ Novosibirsk State Technical University \\ Novosibirsk, Russia \\ odutova@ngs.ru
}

\author{
Shishkin A.V. \\ Dept. of Physical and Chemical Problems of Fuel Energy \\ S.S. Kutateladze Institute of Thermophysics SB RAS \\ Novosibirsk, Russia \\ andrshi@itp.nsc.ru
}

\author{
Shiremetov V.N. \\ Dept. of Electrotechnology \\ Novosibirsk State Technical University \\ Novosibirsk, Russia \\ kaf_aetu@corp.nstu.ru
}

\begin{abstract}
The proposed mathematical models for the calculation of the temperature field and the process of nucleation and growth of a grain in the electrode material of a plasmatron allow conducting numerical simulation of various modes of operation of a hollow cathode and evaluating the change in the structure of a material during electrode heating and using them to study and to improve the operating characteristics of hollow cathodes of vacuum plasmotrons.
\end{abstract}

Keywords- plasmatron, cathode, heat flow, temperature field, recrystallization, structure, grain

\section{INTRODUCTION}

At present, arc plasmatrons are widely used in various fields of science and technology, as well as for solving new electrotechnological problems. The range of plasma-thermal processes, including their application, is increasing all the time. So, the big economic effect allows using plasmatrons on thermal power stations for ignition of coal-dust boilers. Research is actively conducted on the use of plasmatrons in technologies for processing man-caused waste. Because of this, the types of installations used, their capacities, and also the operating modes are very diverse. All this makes the improvement of existing and development of new designs of plasmatrons actual.

The most important element of the vacuum plasmatron is a hollow cathode, which determines all the electrical, power and operational characteristics of a vacuum plasma installation [12].

The use of vacuum plasmatrons with cathodes in the form of a semiclosed cavity made it possible to obtain significant densities of heat fluxes on the heating surface at low operating voltages (30-100 V), as well as allowed obtaining a high efficiency (up to $80-90 \%$ ) in the pressure range from 1 to $10^{3}$ $\mathrm{Pa}[2]$.
The realization of a vacuum arc discharge with a hollow cathode is carried out using a hollow cathode, which is a cylindrical tube with internal diameter $D$, through which gas is fed into a low-pressure chamber. The open end of the tube points toward the anode. The length of the cathode tube should be several of its internal diameters. The rate of destruction of electrodes depends on many factors - the material used, the cooling conditions, the pressure in the discharge chamber, but to a considerable extent, and the parameters of the arc discharge. Therefore, a theoretical and experimental study of the physical-mechanical processes in the material of the plasmatron electrode, aimed at increasing the duration of their operation, is an urgent task. As in many technological processes, the results of experimental studies describe only a part of the phenomena occurring. This makes actual use of the numerical simulation installation in the analysis of multifactor erosion regimes of electrodes. This approach to the study of fundamental processes makes it possible to establish their basic mechanisms, to determine the general patterns from which one can choose both the optimal operating modes for operating and the design of new plasmatrons being developed.

\section{PHYSICAL-MATHEMATICAL MODELING OF PROCESSES OF HEATING HOLLW CATHODES}

The thermal state of the cathode is formed under the influence of plasma on the inner surface of the hollow cathode and, therefore, carries information about these processes and plasma parameters. The peculiarity of the interaction of a plasma with a solid body is the influence on the main characteristics of the interaction not only of the surface of a solid body, but also of processes occurring in its volume. For cathodes of an arc discharge, the electrode temperature field is one of the main quantities characterizing its performance. On the one hand, the emission current density and erosion are determined by the temperature distribution along the working 
surface of the cathode, and on the other hand, the temperature stresses arising from the uneven heating of the electrode can lead to its destruction even before the contact surface with the arc melts. In addition, the mechanical destruction of the electrode can be facilitated by a change in its structure, for example, embrittlement as a result of metal recrystallization, which is important for hollow cathodes [3-5].

It was shown in $[6,7]$ that the position of the currentbinding zone relative to the cathode, i.e. active zone, is determined by the complex interrelation between the electrodynamic and gas dynamic processes occurring in the cathode. It is known from experiments [7] that an increase in the discharge current leads to an increase in the maximum temperature of the cathode and its displacement along the $z$ axis with insignificant changes of active zone range. The current-to-cathode binding zone is localized in a narrow ring, the width $\left(Z_{1}, Z_{2}\right)$ of which increases with current increase.

Let us consider heat transfer in a hollow cathode, which is a cylindrical tube with internal radius $R_{1}$, wall thickness $\delta$ (fig 1). Length $L$ of the cathode tube must be several of its internal diameters. The electrode material is tungsten.

Due to the interaction of the plasma with the inner surface, a conductive heat flux is formed, which determines the temperature field of the cathode as a whole. A total thermal flux within the cavity comes from a conductive heat flux to surface 1 and radiant heat fluxes: radiation flux comes from the cathode cavity through hole 3 and radiation flux of $Q_{c h}$, is absorbed by surface 2 and is taken to the water-cooled cathode arm. Further, a conductive heat flux is dissipated in atmosphere through outer lateral surface $Q_{\text {s }}$ and cathode end surface by radiation of $Q_{f}$ (fig.1).

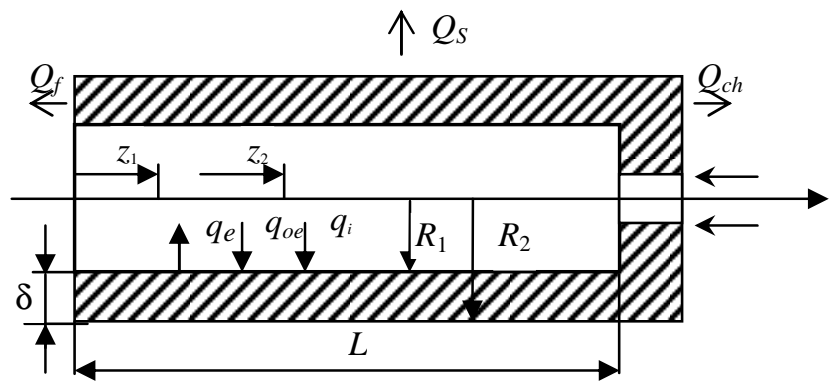

Fig. 1. Thermal exchange circuit in a hollow cathode. $z_{1}, z_{2}-$ coordinates of active zone.

Systematic processing of a large number of experiments [1 - 7] has shown that the dependence of the total heat flux $q_{\Sigma}=q_{i}+q_{o e}-q_{e m}$ on coordinate $\mathrm{z}$ can be specified as Gaussian distribution function $q_{\Sigma}(z)=q_{\max } \exp \left(-\left(z-z_{\max }\right)^{2} / \sigma_{1}\right)$. Parameters $z_{\max }$ are the coordinate of the maximum of heat flux $q_{\max }$, through which the axis of symmetry passes; $\sigma_{1}$ is a parameter.

To determine the temperature field, let us consider the heat equation in the two-dimensional approximation [8]:

$$
c(T) d(T) \frac{\partial T}{\partial t}=\frac{1}{r} \frac{\partial}{\partial r}\left[r \lambda(T) \frac{\partial T}{\partial r}\right]+\frac{\partial}{\partial z}\left[\lambda(T) \frac{\partial T}{\partial z}\right]+q_{j},
$$

where $c, d, \lambda, \rho$ - specific heat, density, thermal conductivity coefficient, specific resistance of the material; $q_{j}=j_{0}{ }^{2}(r$, $z) \rho(T) ; j_{0}(r, z)$ is the current density distribution in the electrode, which is determined from the solution of the Laplace equation, which characterizes the distribution of potential $\varphi$ in the cathode wall:

$$
\frac{1}{r} \frac{\partial}{\partial r}\left[r \frac{1}{\rho(T)} \frac{\partial \varphi}{\partial r}\right]+\frac{\partial}{\partial z}\left[\frac{1}{\rho(T)} \frac{\partial \varphi}{\partial z}\right]=0
$$

From found field $\varphi(r, z)$, the current density is determined by $j_{0}$ by the formula:

$$
j_{0}=\frac{1}{\rho(T)}\left[\left(\frac{\partial \varphi}{\partial r}\right)^{2}+\left(\frac{\partial \varphi}{\partial z}\right)^{2}\right]^{1 / 2}
$$

The boundary conditions for equation (1) are as follows: On the inner surface:

$$
-\lambda \frac{\partial T\left(R_{1}, z\right)}{\partial r}=q_{\Sigma}(z), \quad 0 \leq z \leq L .
$$

On the outer surface - radiation according to the StefanBoltzmann law:

$$
-\lambda \frac{\partial T\left(R_{2}, z\right)}{\partial r}=-\varepsilon(T) \sigma T^{4}\left(R_{2}, z\right), \quad 0 \leq z \leq L,
$$

where $\varepsilon$ is the degree of blackness of the material; $\sigma$ is the Stefan-Boltzmann constant;

At the end surface of the cathode, losses due to radiation are taken into account:

$$
-\lambda \frac{\partial T(r, 0)}{\partial z}=-\varepsilon(T) \sigma T^{4}(r, 0), \quad R_{1} \leq r \leq R_{2}
$$

At the boundary with the cathode holder, an ideal thermal contact is assumed:

$$
T(r, L)=T_{k d}, \quad R_{1} \leq r \leq R_{2},
$$

where $T_{k d}$ is the temperature of the cathode holder.

The initial condition is:

$$
T(r, z, t)=T_{0}
$$

The boundary conditions for equation (2) are as follows: On the inner surface in the zone of current binding to the cathode:

$$
-\frac{1}{\rho} \frac{\partial \varphi\left(R_{1}, z\right)}{\partial r}=j_{\Sigma}(z), \quad 0 \leq z \leq L .
$$

On the outer surface: 


$$
\frac{\partial \varphi\left(R_{2}, z\right)}{\partial r}=0, \quad 0 \leq z \leq L
$$

At the end and at the boundary with the cathode holder:

$$
\frac{\partial \varphi(r, 0)}{\partial z}=0, \quad \varphi(r, L)=0, \quad R_{1} \leq r \leq R_{2}
$$

To solve the problem by the method of finite differences, the authors used a second-order scheme for approximating with respect to spatial coordinates and the first-order scheme with respect to time [9]. The resulting difference equations were solved by the sweep method.

Calculations were carried out for heat flows with parameters:

$$
\begin{aligned}
& \text { a) } \quad q_{\max }=2.5 \quad \mathrm{MW} / \mathrm{m}^{2}, \quad z_{\max }=0.02027 \quad \mathrm{~m}, \quad \sigma_{1}=0.0004, \\
& j_{\Sigma}(z)=9 \cdot 10^{5} \exp (-(z-0.03) 2 / 0.0002) ; \\
& \text { b) } q_{\max }=3.5 \quad \mathrm{MW} / \mathrm{m}^{2}, \quad z_{\max }=0.02027 \mathrm{~m}, \quad \sigma_{1}=0.00022, \\
& j_{\Sigma}(z)=1.4 \cdot 10^{6} \exp \left(-\left(z-z_{\max }\right)^{2} / \sigma_{1}\right) .
\end{aligned}
$$

Thermophysical properties of the material depend on temperature.

\section{RESULT AND DISCUSSION}

Heating the cathode is characterized by high speeds and a fairly fast output to the stationary regime. Already from the time $t \sim 50-55 \mathrm{~s}$, the temperature of all the points changes insignificantly (Figure 2). Fig. 3. gives an idea of the stationary thermal state of the cathode. One can note a sharp change in temperature along the length of the electrode in the vicinity of the active zone (the heated surface) and a smooth change in the remaining parts of the structure. The temperature drop across the thickness of the electrode does not exceed $\sim 100-200 \mathrm{~K}$. As seen from the graphs (Fig. 2-3), large axial and radial temperature gradients arise in the cathode material. Hence, the electrode is not only under the influence of large thermal loads, but also under significant thermomechanical stresses. When the arc is applied to the surface of the electrode already at the beginning of the heating process, an intensive volumetric expansion of the metal occurs in the arc-affected zone, and the intensity and magnitude of the expansion are determined by the rate and temperature of heating. The increase in volume is prevented by cold layers surrounding the heating region, resulting in the development of compressive stresses in the zone of temperature influence, the magnitude of which is greater, the higher the metal heating temperature. Stretching stresses can exceed the tensile strength and contribute to the formation of microcracks. In the future, all this can cause changes in the temperature distribution (increase in surface temperature, its evaporation, increase in erosion).

A comparison of the position of the current-binding zone with the temperature distribution over the surface shows that the maximum temperature of the cathode wall is in the current-binding zone, and the length of the high-temperature zone is much longer than its length.

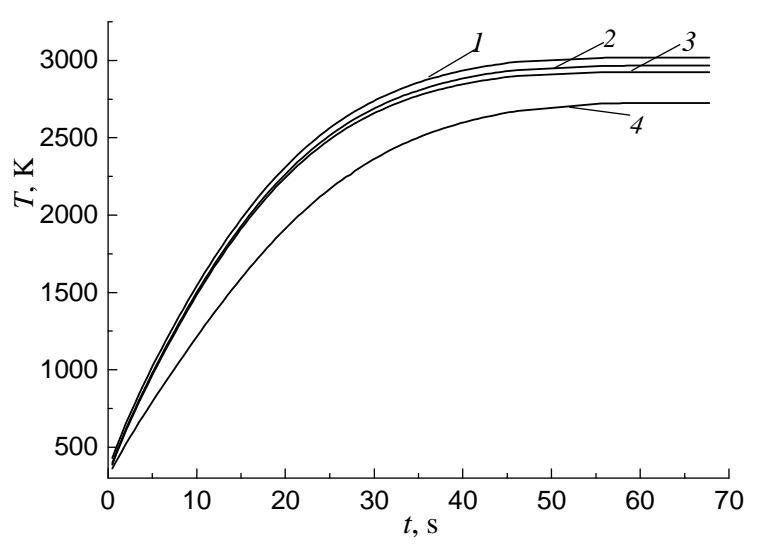

Fig. 2. The temperature changes of different points with time: (qmax $=2.5$ $\left.\mathrm{MW} / \mathrm{m}^{2}\right) r=R_{1}$ and $z=z_{\text {max }}(1) ; R=R_{1}+\delta / 2, z=z_{\max }(2) ; R=R_{2}, z=z_{\max }$ (3); $R=R_{1}$ and $z=L / 2$ (4).

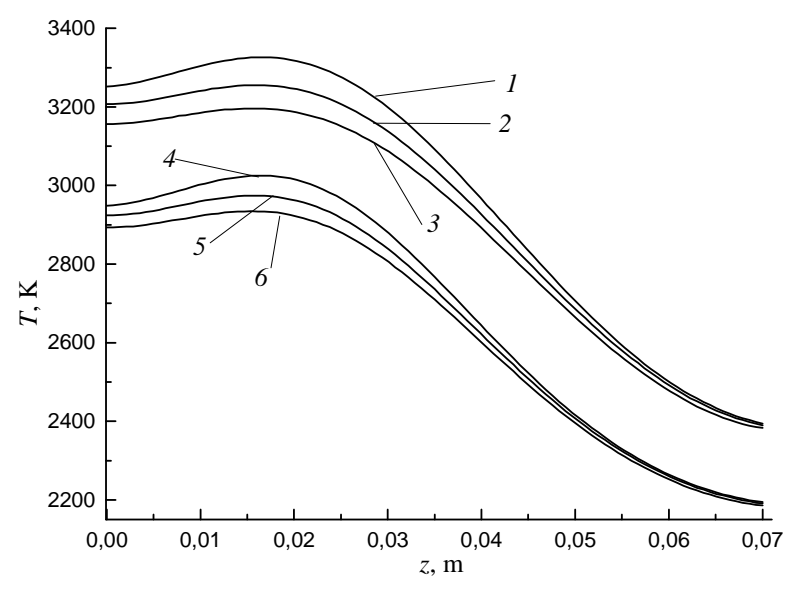

Fig. 3. Temperature distribution along the length of the electrode at time $t=$ $t_{k}$ : (1), (4) - $r=R_{1} ;(2),(5)-r=R_{1}+\delta / 2 ;(3),(6)-r=R_{2} ; q_{\max }=3.5 \mathrm{MW} / \mathrm{m}^{2}$ $(1,2,3)$ and $q_{\max }=2.5 \mathrm{MW} / \mathrm{m}^{2}$.

\section{DEPENDENCE OF GRAIN GROWTH ON TEMPERATURE IN THE MATERIAL OF THE HOLLOW CATHODE}

A polycrystal consists of a large number of grains with differently oriented crystal lattices. Intergranular boundaries (IGB) are called large-angle boundaries. Crystallographic directions in neighboring grains form angles reaching tens of degrees. The large-angle boundaries are a transition layer 1-5 $\mathrm{nm}$ in width. In it, the correct arrangement of atoms is disrupted, and the concentration of impurities is increased.

Each grain consists of individual subgrains forming a substructure. The subgrain is disoriented relative to each other from several parts to units of degrees $(<5)$ - so there are smallangle boundaries. 
In accordance with the wavelength of an electron in a metal, the main scattering of electrons occurs on point defects, which are especially numerous near the IGB. Therefore, the area of the IGB, and consequently the grain size, is of the greatest interest since they have a significant effect on the electrical resistivity and thermal conductivity.

When the plasmatron electrode is operating, the process of formation and growth of new grain nuclei takes place. The temperature dependence of parameters $N$ - rate of nucleation of undeformed grains and $G$ - rate of their growth can be expressed with the help of equations [10]:

$$
\begin{aligned}
& G=K_{G} \exp \left(-\Delta H_{G} / R T\right) ; \\
& N=K_{N} \exp \left(-\Delta H_{N} / R T\right),
\end{aligned}
$$

where $K_{G}, K_{N}$ are multipliers; $\Delta H_{G}$ - activation energy in the processes of grain growth; $\Delta K_{N}$ - activation energy in the processes of nucleation of grains; $R=8.314 \mathrm{~J} /(\mathrm{K} \mathrm{mol})$ is the universal gas constant. Based on the data of [11] for tungsten fiberboard, $G=10^{15} \exp \left(-753 \cdot 10^{3} / R T\right)$. The rate of nucleation in recrystallization in accordance with (5) has an activation mechanism in accordance with (5). Therefore, for calculation by analogy, one can use the formula for the growth of a new phase nucleus, suggesting that the latent heat of formation is equivalent in this case to the activation energy of primary recrystallization [12]:

$N_{\text {гет }}=\frac{16 \pi n^{\prime} D_{0}}{a^{4}}\left(\frac{\gamma T_{p}}{\rho \kappa \Delta T}\right)^{2} \exp \left[-\frac{E_{D}+(16 / 3) \pi \gamma^{3}\left(T_{\mathrm{p}} / \rho \kappa \Delta T\right)^{2}}{k_{\mathrm{B}} \cdot\left(T_{\mathrm{p}}+\Delta T\right)}\right]$

where $n^{\prime}=0.217 n_{s(100)} S / V=0.217 n_{s(100)} 3 / r_{\text {drec }}$ - number of atoms on the surface of the precrystallization grain, referred to its volume; $S$ - grain boundary area, $V$ - grain volume; $r_{\text {drec }}$ is the average grain radius before recrystallization; $n_{\mathrm{s}(100)}{ }^{-}$ number of atoms per unit surface of the face (100) of the bcc crystal; $D_{0}$ is the pre-exponential factor in the temperature dependence of the diffusion coefficient; $a$ - parameters of the tungsten lattice; $\gamma$ is the energy of the surface; $T_{\mathrm{p}}$ is the temperature of recrystallization; $k$ - specific activation energy of recrystallization; $\Delta T$ is the superheat temperature; $E_{D}$ is the activation energy of diffusion; $k_{\mathrm{B}}=1.38 .10^{-23}(\mathrm{~J} / \mathrm{K})$ is the Boltzmann constant.

The time of complete primary recrystallization is $\tau_{R}=\left(\pi \cdot G^{2} \cdot N\right)^{-1 / 3}$. The average grain diameter in the direction of crystallization is $D(\Delta T)=2 G(\Delta T) \cdot \tau_{R}=2\left(G /(\pi N)^{1 / 3}\right.$.

Three interrelated processes are the most essential for the determination of primary recrystallization. This is the process of heating, the process of nucleation and the process of growth of undeformed grain. The results of the calculation are shown in Fig. 4 - 5. It is possible to see that in the region of 300-400 $\mathrm{K}$, the grain size has a maximum (Fig. 4).

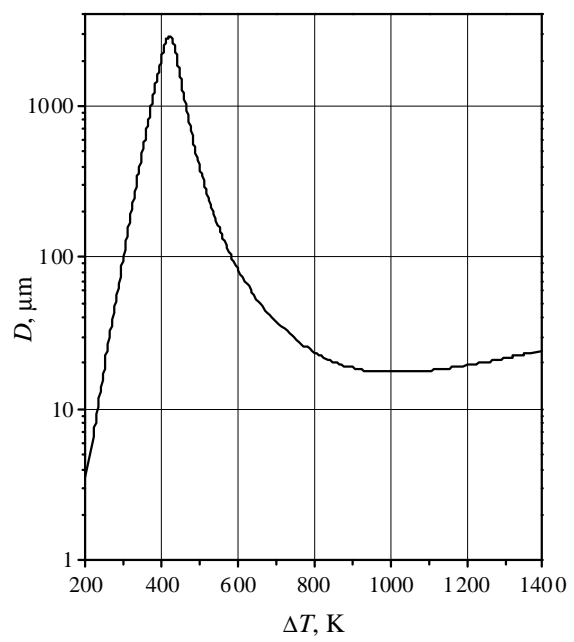

Fig. 4. Dependence of the size of recrystallized grain on overheating

This is because at these differences, the rate of grain formation begins to outrun the rate of its growth and the grain begins to decrease in size (Fig. 5).

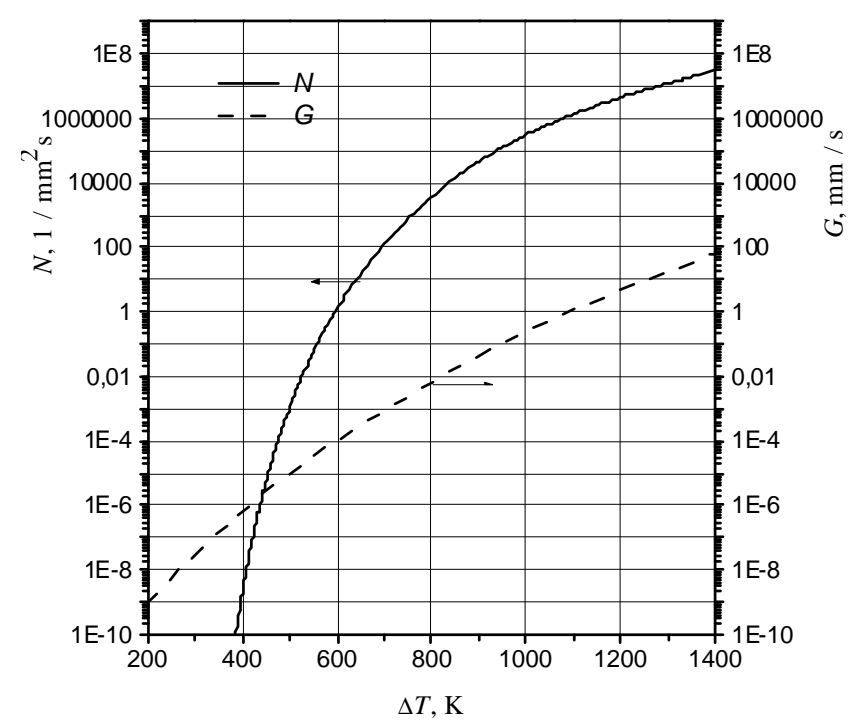

Fig. 5. Dependence of formation rate and grain growth on overheating

Figs. 6 - 7 show the distribution of the size of the recrystallized grain along the radius and the length of active zone of the hollow cathode for flows $q_{\max }=3.5 \mathrm{MW} / \mathrm{m}^{2}$. For $t=t_{k}$ and $z=10 \mathrm{~mm}$, the grain size decreases since for the given region, the rate of nucleation exceeds the rate of grain growth (Fig. 6). 


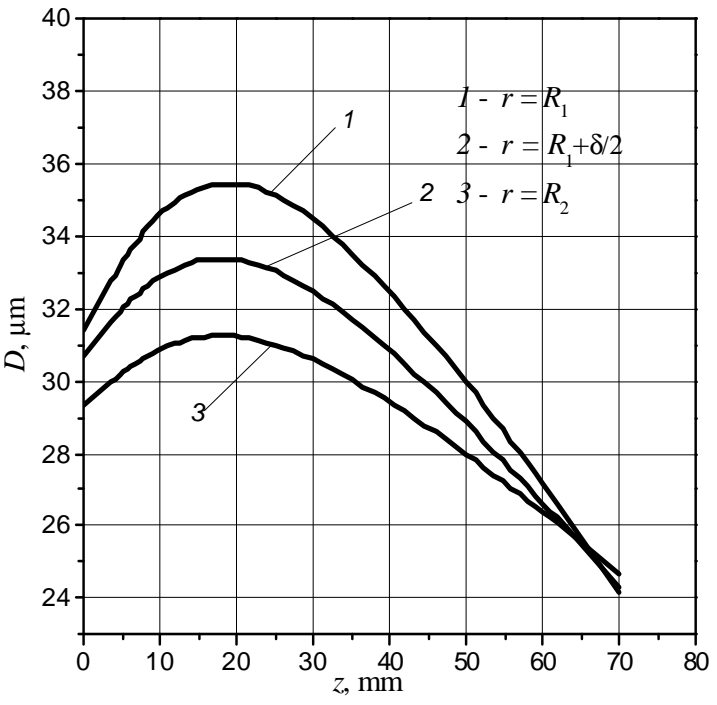

Fig. 6. Distribution of the size of the recrystallized grain along the radius of the cathode

The graph of the distribution of grain growth along the length of the active zone of the cathode (Figure 7) shows that the grain decreases in the colder parts of the cathode and increases in hotter areas. The grain size for the final sections of active zone of the hollow cathode tends to 0 . This is because at low times, the recrystallization process is very slow or generally does not take place, because the heating temperature does not reach the recrystallization temperature.

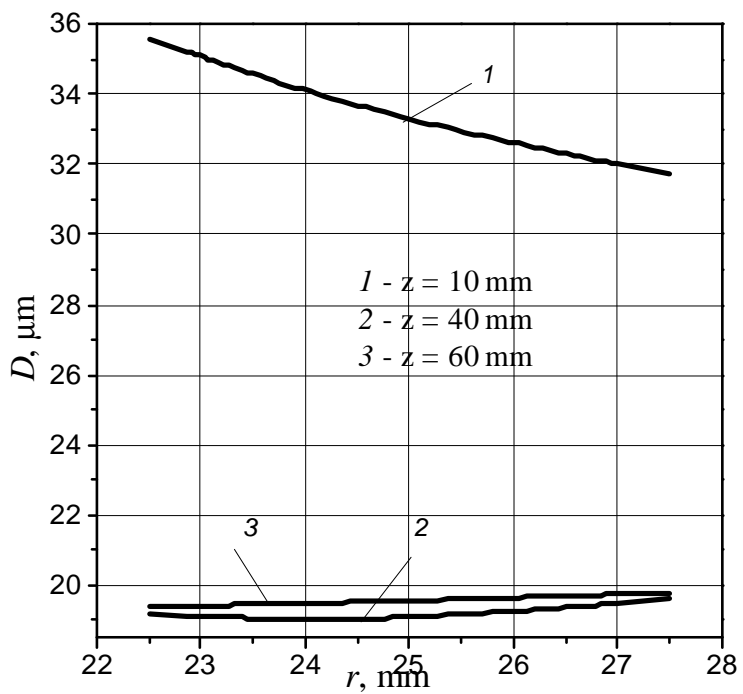

Fig. 7. Distribution of the size of the recrystallized grain along the length of the active zone of the cathode

\section{CONCLUSION}

The solutions obtained showed that the heating of the cathode is characterized by high velocities and a fairly fast output to the stationary regime. One should note a sharp change in temperature along the length of the electrode in the vicinity of the active zone (the heated surface). A characteristic feature of the temperature distribution is the large axial and radial temperature gradients that can lead to large thermal stresses in the body of the electrode. In addition, the mechanical destruction of the electrode can also contribute to a change in its structure as a result of the recrystallization of the metal.

\section{References}

[1] V.S. Cherednichenko, M.V.Cherednichenko,Vacuum plasma electric furnaces with hollow cathodes. Tutorial. Novosibirsk: Publishing House of the National Technical University, 1999.

[2] V.S. Cherednichenko, A.S. Anshakov, and M.G Kuzmin, Plasma electrotechnological installations. Novosibirsk: Publishing House of the National Technical University, 2005.

[3] J.Devautor, J.P. Chabrerie, $\mathrm{Ph}$ Teste, The study of the thermal processes in an electro- genic is presented to the lecture arc, J. Phys. III France, vol. 3, pp. 1157-1166, 1993.

[4] Ph.Teste, T.Leblanc, J.P Chabrerie, Study of the arc root displacement and three-dimensional modulation of the thermal phenomena occurring in hollow cathode submitted to an electric moving arc, J. Phys. D: Appl. Phys, vol. 28, pp. 888-898, 1995.

[5] P.Solana, Ph.Kapadia, J. A Dowden, Mathematical analysis of the heating effects and electrode erosion in conical arc cathode, J. Phys. D: Appl. Phys, vol. 31, pp. 3446-3456, 1998.

[6] V.S. Cherednichenko, High-current vacuum arcs with a hollow cathode. The thermal field of a cathode. Izv. SB AS USSR. A series of Technical Sciences, № 7, issue 2, pp. 91-96,1987.

[7] V.S. Cherednichenko, S.G. Galkin, V.A. Kosinov, High-current arcs with a hollow cathode, Generation of streams of electric arc plasma, Novosibirsk: Institute of Thermal Physics, Siberian Division, USSR Academy of Sciences, 1987, pp. 306-322.

[8] A.M. Zimin, I.P. Nozarenko, I.G. Panevin, Low-temperature plasma (10). Mathematical modeling of cathode processes, Novosibirsk: Science. Siberian Publishing Company, 1993.

[9] A.A. Samarsky, Introduction to numerical methods, Moscow: Nauka, 1982.

[10] S.S. Gorelik, Recrystallization of metals and alloys, M., 1967.

[11] E.I. Zasimchuk, V.I. Isaichev, Kinetics of primary, secondary and collective recrystallization of a tungsten wire, Metallophysics, Moscow: Naukova Dumka, 1970, issue 31.

[12] V.P. Saburov, A.N. Cherepanov, M.F. Zhukov, Low-temperature plasma (12). Plasma-chemical synthesis of ultradisperse powders and their application, Novosibirsk: Science. Siberian Publishing Company RAS, 1995. 